\title{
Relações entre precipitações intensas de diferentes durações ocorridas no município de Urussanga, SC
}

\begin{abstract}
$\overline{\text { Álvaro J. Back }}$
RESUMO

Neste trabalho se analisaram os pluviogramas do período de 1981 a 2004 da estação meteorológica de Urussanga, SC (Latitude $28^{\circ} 31^{\prime} \mathrm{S}$, longitude $49^{\circ} 19^{\prime} \mathrm{W}$, altitude $49 \mathrm{~m}$ ). A partir das séries de chuvas máximas anuais foram estimadas, por meio da distribuição de Gumbel-Chow, as chuvas máximas com durações variando de 5 min a 24 h, e também para a duração de 1 dia considerando-se os períodos de retorno de 2, 5, 10, 20, 25, 50 e 100 anos. A aderência dos dados ao modelo de Gumbel-Chow foi comprovada através do teste de Kolmogorov-Smirnov, com nível de significância de 5\%. A razão entre a precipitação máxima de 24 h e a precipitação máxima de 1 dia variou de 1,17 para o período de retorno de 2 anos até 1,11 para o período de retorno de 100 anos, com média de 1,13. Ajustaram-se os coeficientes da equação proposta por Bell a fim de estimar a relação entre a chuva com duração t min e período de retorno T anos, em função da chuva máxima com período de retorno de 10 anos e duração de $1 \mathrm{~h}$. Do mesmo modo, se ajustaram também os coeficientes da equação de Bell cuja base foi a chuva máxima com período de retorno de 10 anos e duração de 1 dia.
\end{abstract}

Palavras-chave: chuvas intensas, hidrologia, drenagem

\section{Relationships between heavy precipitations of different durations in the municipality of Urussanga, SC}

\begin{abstract}
Recorded rainfalls in the period from 1981 to 2004 at the meteorological station of Urussanga, SC (Latitude $28^{\circ} 31^{\prime}$ S, longitude $49^{\circ} 19^{\prime} \mathrm{W}$, altitude $49 \mathrm{~m}$ ), were analyzed. From the series of annual maximum precipitation, the maximum rainfalls were estimated using the Gumbel-Chow distribution, with durations varying from 5 min to $24 \mathrm{~h}$ and also for the duration of one day, considering the return periods of 2, 5, 10,20, 25, 50 and 100 years. The adherence of the data to the Gumbel-Chow model was proven through the Kolmogorov-Smirnov test with a significance level of $5 \%$. The ratio between the $24 \mathrm{~h}$ maximum precipitation and the maximum precipitation of one day varied from 1.17 to 2 years return period up to 1.11 for the 100 years return period, with average of 1.13 . The coefficients of the equation proposed by Bell were adjusted to estimate the relationship between the rain with duration of $\mathrm{t}$ min and return period $\mathrm{T}$ years as a function of the maximum rain with a return period of 10 years and duration of $1 \mathrm{~h}$. In the same way, the coefficients of the Bell equation were adjusted based on the maximum rain with return period of 10 years and duration of 1 day.
\end{abstract}

Key words: heavy rainfall, hydrology, drainage 


\section{INTRODUÇÃO}

O dimensionamento dos projetos hidráulicos que envolvem o escoamento superficial é realizado com base na intensidade máxima de chuva com determinado risco de ser igualada ou superada, definido pelo período de retorno. Normalmente, as estimativas das intensidades de chuva são realizadas com base nas relações Intensidade-Duração-Freqüência (IDF) de chuvas. Nesse sentido se procura ajustar, para os locais com registros pluviográficos, as equações de chuvas intensas, também chamadas equações IDF, destacandose os trabalhos de Pinto et al. (1996) no Estado de Minas Geais, Fendrich (1998) no Estado do Paraná, Costa \& Brito (1999) e Oliveira et al. (2000) no Estado de Goiás, Martinez Junior (1999) no Estado de São Paulo, Silva et al. (1999) para o Estado do Rio de Janeiro e Espírito Santo, Silva et al. (2002) para o Estado da Bahia, e Back (2002) e Nerilo et al. (2002) para o Estado de Santa Catarina.

A dificuldade na obtenção das equações IDF de chuvas intensas reside na escassez de registros pluviográficos e/ou nos pequenos períodos de observação. Souza \& Silva (1998) apresentaram um método analítico e alternativo para estimar a intensidade máxima de precipitação a partir da curva normalizada de precipitação. Algumas metodologias foram desenvolvidas para obtenção das chuvas de menor duração, a partir dos dados pluviométricos diários. Torrico (1975) desenvolveu a metodologia das isozonas, que pode ser aplicada em todo o território nacional. Damé (2001) e Damé et al. (2003) utilizaram séries sintéticas de precipitação para a estimativa das relações IDF mas, dentre os métodos mais usados, se destacam aqueles que se baseiam nas relações entre precipitações de diferentes durações para desagregar a chuva máxima diária em chuva com duração inferior; trata-se de um método com a vantagem de ser de uso simples, de fornecer resultados satisfatórios e com grande similaridade para diferentes locais, o que lhe outorga validade regional.

Bell (1969) estabeleceu relações empíricas entre precipitações com diferentes durações baseadas em dados de séries parciais de chuva observada nos EUA, Austrália, URSS, Porto Rico, Alasca, África do Sul e Havaí. O fundamento teórico desse estudo é a existência de similaridade entre os mecanismos das tormentas. O valor máximo dessas chuvas está associado a células convectivas, que têm características semelhantes em muitas partes do mundo e, por isso mesmo, se utiliza esta equação para estimar as precipitações máximas entre os limites especificados. Este trabalho é bastante clássico dentro deste tema, mas apresenta limitações, entre elas o fato de que suas equações foram obtidas a partir de dados de chuva de diversas partes do mundo sendo, portanto, seus resultados, função de valores médios e não específicos para um local; outra limitação é que o valor da precipitação máxima obtida é válido apenas para durações entre 5 e $120 \mathrm{~min}$; pode-se, também destacar, como limitação ao método de Bell, a necessidade de se conhecer a chuva máxima com duração de $1 \mathrm{~h}$ e período de retorno de 10 anos, fato que requer dados de estação com pluviógrafo. Mello et al. (2003) ajustaram o método de Bell para diferentes regiões do Estado de Minas Gerais tomando como base a chuva máxima com duração de 1 h e período de retorno de 2 anos.

O ajuste da equação de Bell tendo como base a chuva máxima com duração de 1 dia e período de retorno de 10 anos, permite que se obtenha, em um único procedimento, a altura máxima da chuva em uma duração qualquer sem a necessidade de utilização de outros coeficientes regionais. Propôs-se, então, com este trabalho, ajustar as equações que relacionam as precipitações com diferentes durações aos dados de Urussanga e compará-las com as estimativas das chuvas obtidas a partir da chuva máxima com duração de 1 dia.

\section{MATERIAL E MÉTODOS}

Utilizaram-se os gráficos diários do pluviógrafo da Estação Meteorológica de Urussanga, SC (latitude 28 31' S, longitude $49^{\circ} 19^{\prime} \mathrm{W}$, altitude $49 \mathrm{~m}$ ) do período de 1981 a 2006 e se determinaram, após a digitalização dos pluviogramas, as séries de máximas anuais de chuva com duração de 5, 10, $15,20,25,30,45,50,55,60,75,90$ e $105 \min$ e 2, 3, 4 , $6,7,8,10,12,14,16,18,20,22$ e 24 h, além da chuva máxima de 1 dia, definida como o total da chuva acumulada diariamente até as $9 \mathrm{~h}$.

Para cada duração foram estimados, também, os valores de chuva com período de retorno de 2, 5, 10, 20, 25, 50 e 100 anos, por meio da distribuição de Gumbel-Chow, como:

$$
P_{T}=\bar{x}+\left(Y-Y_{n}\right) \frac{S}{S_{n}}
$$

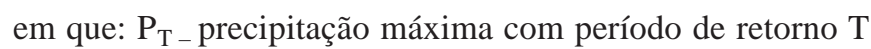
anos, $\mathrm{mm}$

X - média da série de máximas anuais, mm

$\mathrm{S}$ - desvio padrão da série de máximas anuais, mm

Yn - média da variável reduzida

Sn - desvio padrão da variável reduzida

Y - variável reduzida calculada por:

$$
\mathrm{Y}=-\ln \left\{-\ln \left(1-\frac{1}{\mathrm{~T}}\right)\right\}
$$

em que T é o período de retorno (anos).

A aderência das séries de máximas anuais à distribuição da Gumbel-Chow, foi testada pelo teste de KolmogorovSmirnov a nível de significância de 5\%, conforme descrito por Kite (1978).

Ajustaram-se, com as alturas das chuvas estimadas, os coeficientes da equação de Bell em função da chuva máxima com período de retorno de 10 anos e duração de $1 \mathrm{~h}$ (60 min) $\left(\mathrm{P}_{10}{ }^{60}\right)$, com a seguinte apresentação:

$$
\mathrm{P}_{\mathrm{T}}^{\mathrm{t}}=(\mathrm{aLn}(\mathrm{T})+\mathrm{b})\left(\mathrm{ct}^{\mathrm{d}}-\mathrm{e}\right) \mathrm{P}_{10}^{60}
$$

em que: a, b, c, d, e - coeficientes empíricos

$\mathrm{P}_{\mathrm{T}}^{\mathrm{t}}$ - precipitação estimada com período de retorno $\mathrm{T}$ anos e duração t min;

$\mathrm{P}_{10}^{60}$ - precipitação observada com período de retorno de 10 anos e duração de 60 min. 
Os valores obtidos foram comparados com a equação proposta por Bell (1969) dada por:

$$
\mathrm{P}_{\mathrm{T}}^{\mathrm{t}}=(0,21 \operatorname{Ln}(\mathrm{T})+0,52)\left(0,54 \mathrm{t}^{0,25}-0,5\right) \mathrm{P}_{10}^{60}
$$

e a equação proposta por Uehara et al. (1980) para o Brasil e São Paulo com respectivas expressões:

$$
\begin{aligned}
& \mathrm{P}_{\mathrm{T}}^{\mathrm{t}}=(0,1824 \operatorname{Ln}(\mathrm{T})+0,58)\left(0,497 \mathrm{t}^{0,27}-0,5\right) \mathrm{P}_{10}^{60} \\
& \mathrm{P}_{\mathrm{T}}^{\mathrm{t}}=(0,22 \operatorname{Ln}(\mathrm{T})+0,50)\left(0,38 \mathrm{t}^{0,31}-0,39\right) \mathrm{P}_{10}^{60}
\end{aligned}
$$

Realizou-se, também, o ajuste dos coeficientes da equação de Bell, em função da chuva máxima com período de retorno de 10 anos e duração de 1 dia, dada por:

$$
\mathrm{P}_{\mathrm{T}}^{\mathrm{t}}=(\mathrm{aLn}(\mathrm{T})+\mathrm{b})\left(\mathrm{ct}^{\mathrm{d}}-\mathrm{e}\right) \mathrm{P}_{10}^{1 \mathrm{dia}}
$$

em que: $\mathrm{P}_{10}^{1 \mathrm{dia}}$ - precipitação estimada com período de retorno de 10 anos e duração de 1 dia.

$\mathrm{O}$ ajuste dos coeficientes foi realizado minimizando-se a função S, dada por:

$$
\mathrm{S}=\sum_{\mathrm{t}=1}^{\mathrm{i}} \sum_{\mathrm{T}=1}^{\mathrm{J}}\left(\mathrm{G}_{\mathrm{T}}^{\mathrm{t}}-\mathrm{P}_{\mathrm{T}}^{\mathrm{t}}\right)^{2}
$$

em que:

S - Somatório dos quadrados dos desvios entre a precipitação estimada pela equação 3 ou 4, e a precipitação observada

$\mathrm{t}$ - duração da chuva, min

$\mathrm{T}$ - período de retorno, anos

G - precipitação estimada pela distribuição GumbelChow para duração t e período de retorno $\mathrm{T}$.

Equações gerais para duração de 5 min até 24 h (1440 min) foram ajustadas e comparadas com equações ajustadas para duração de 5 a $120 \mathrm{~min}$, de 120 a $480 \mathrm{~min}$ e de 480 a 1440 min.

Para avaliar a precisão das estimativas utilizou-se o erro padrão de estimativa, dado por:

$$
E p=\sqrt{\frac{\left(G_{T}^{T}-P_{T}^{t}\right)^{2}}{N}}
$$

em que:

Ep - erro padrão de estimativa, mm
$\mathrm{N}$ - número de observações.

\section{RESULTADOS E DISCUSSÃO}

Na Tabela 1 se encontram os valores de precipitação estimada para as diversas durações e períodos de retorno; constam, também, as relações entre precipitação máxima com duração de 24 h sobre a precipitação máxima de 1 dia, observando-se que essas relações variam de 1,17 para o período de retorno de 2 anos a 1,11 para o período de retorno de 100 anos, com valor médio de 1,13. Esses valores confirmam as observações de Genovez et al. (1994) de que as relações entre precipitação máxima em 24 h e a precipitação de 1 dia, são praticamente constantes e independentes do período de retorno.

Na desagregação da chuva diária o primeiro passo é a obtenção da chuva máxima em 24 h; já com os dados dos pluviômetros se obtém as chuvas máximas de 1 dia, que diferem das máximas em 24 h, em virtude das leituras serem realizadas sempre no mesmo horário e, portanto, podem, em alguns eventos, ser inferiores ao máximo em 24 h. Vários trabalhos mostram que a proporção entre a chuva máxima em 24 h e a chuva máxima de 1 dia é praticamente constante e independente do período de retorno. Occhipinti \& Santos (1966) utilizando séries de dados de 1928 a 1965 de São

\begin{tabular}{|c|c|c|c|c|c|c|c|}
\hline \multirow{2}{*}{$\begin{array}{c}\text { Duração } \\
\text { (min) }\end{array}$} & \multicolumn{7}{|c|}{ T- Período de Retorno (anos) } \\
\hline & 2 & 5 & 10 & 20 & 25 & 50 & 100 \\
\hline 5 & 10,1 & 12,5 & 14,1 & 15,7 & 16,2 & 17,7 & 19,2 \\
\hline 10 & 16,0 & 19,6 & 21,9 & 24,2 & 24,9 & 27,2 & 29,4 \\
\hline 15 & 21,3 & 27,2 & 31,0 & 34,8 & 36,0 & 39,6 & 43,2 \\
\hline 20 & 24,5 & 32,0 & 37,0 & 41,8 & 43,3 & 47,9 & 52,5 \\
\hline 25 & 26,7 & 35,8 & 41,9 & 47,6 & 49,5 & 55,1 & 60,8 \\
\hline 30 & 28,6 & 38,9 & 45,7 & 52,2 & 54,3 & 60,6 & 66,9 \\
\hline 35 & 30,2 & 41,3 & 48,7 & 55,7 & 58,0 & 64,9 & 71,7 \\
\hline 40 & 31,4 & 43,5 & 51,4 & 59,1 & 61,5 & 69,0 & 76,4 \\
\hline 45 & 32,7 & 45,5 & 53,9 & 62,1 & 64,6 & 72,6 & 80,4 \\
\hline 50 & 33,8 & 46,8 & 55,4 & 63,7 & 66,3 & 74,3 & 82,4 \\
\hline 55 & 34,8 & 47,7 & 56,3 & 64,6 & 67,2 & 75,2 & 83,2 \\
\hline 60 & 35,7 & 48,8 & 57,5 & 65,8 & 68,5 & 76,6 & 84,7 \\
\hline 75 & 37,8 & 51,3 & 60,3 & 68,9 & 71,7 & 80,1 & 88,5 \\
\hline 90 & 39,8 & 53,9 & 63,2 & 72,2 & 75,0 & 83,8 & 92,5 \\
\hline 105 & 41,6 & 56,1 & 65,7 & 74,9 & 77,8 & 86,8 & 95,7 \\
\hline 120 & 43,5 & 57,9 & 67,4 & 76,5 & 79,4 & 88,3 & 97,1 \\
\hline 150 & 46,4 & 61,9 & 72,1 & 81,9 & 85,0 & 94,6 & 104,1 \\
\hline 180 & 48,7 & 65,0 & 75,9 & 86,3 & 89,5 & 99,7 & 109,8 \\
\hline 240 & 53,0 & 72,4 & 85,2 & 97,5 & 101,4 & 113,4 & 125,3 \\
\hline 300 & 56,8 & 77,7 & 91,5 & 104,8 & 109,0 & 121,9 & 134,8 \\
\hline 360 & 60,7 & 84,5 & 100,2 & 115,3 & 120,0 & 134,8 & 149,4 \\
\hline 420 & 64,8 & 91,5 & 109,3 & 126,3 & 131,7 & 148,3 & 164,8 \\
\hline 480 & 68,1 & 97,0 & 116,1 & 134,4 & 140,2 & 158,2 & 176,0 \\
\hline 600 & 73,1 & 104,9 & 126,0 & 146,2 & 152,6 & 172,3 & 191,9 \\
\hline 720 & 77,9 & 113,6 & 137,1 & 159,8 & 166,9 & 189,0 & 211,0 \\
\hline 840 & 82,0 & 118,9 & 143,4 & 166,8 & 174,3 & 197,2 & 219,9 \\
\hline 960 & 85,8 & 124,4 & 149,9 & 174,4 & 182,2 & 206,2 & 229,9 \\
\hline 1080 & 89,2 & 128,4 & 154,3 & 179,2 & 187,1 & 211,4 & 235,5 \\
\hline 1200 & 91,9 & 131,8 & 158,3 & 183,6 & 191,7 & 216,5 & 241,1 \\
\hline 1320 & 94,8 & 134,7 & 161,1 & 186,4 & 194,4 & 219,1 & 243,7 \\
\hline 1440 & 97,5 & 138,2 & 165,2 & 191,0 & 199,2 & 224,5 & 249,5 \\
\hline 1 dia & 83,2 & 121,3 & 146,5 & 170,7 & 178,3 & 202,0 & 225,4 \\
\hline$P_{24 h} / P_{1 \text { dia }}$ & 1,17 & 1,14 & 1,13 & 1,12 & 1,12 & 1,11 & 1,11 \\
\hline
\end{tabular}
Paulo observaram a relação média de 1,14.

Genovez \& Pegoraro (1998) analisando dados de 23 pluviógrafos do Estado de São Paulo, obtiveram valor mínimo de 1,03 e máximo de 1,17 com média de 1,13. Baseada em

Tabela 1. Precipitação máxima estimada 
dados de vários pluviógrafos do Brasil, a CETESB (1986) recomendou o valor 1,14. Segundo Froehlich (1993), o U. S. Weather Bureau indicou o valor de 1,13 e Kessler \& Raad (1978) recomendaram o valor de 1,10. Weiss (1964), com base em conceitos teóricos de probabilidade, estudou as relações entre precipitações médias obtidas por médias móveis e as precipitações obtidas em intervalos fixos e obteve a relação de 1,14 .

Na Tabela 2 se encontram os coeficientes das equações, ajustados para a estimativa da relação da chuva com período de retorno $\mathrm{T}$ e duração t, em função da chuva estimada com período de retorno de 10 anos e duração de 60 min $\left(\mathrm{P}_{10}{ }^{60}\right)$. Obteve-se, com a equação geral, erro padrão de estimativa da chuva de $4,8 \mathrm{~mm}$, reduzindo para valores abaixo de $2,6 \mathrm{~mm}$, quando as equações são ajustadas para intervalos menores.

Tabela 2. Coeficientes da equação de Bell em função da chuva com período de retorno de 10 anos e duração de $60 \min \left(\mathrm{P}_{10}{ }^{60}\right)$

\begin{tabular}{ccccccc}
\hline $\begin{array}{c}\text { Duração } \\
\text { (min) }\end{array}$ & a & b & c & d & e & $\begin{array}{c}\text { Erro padrão } \\
(\mathbf{m m})\end{array}$ \\
$5-120$ & 1,356 & 3,220 & 1,342 & 0,032 & 1,378 & 2,2 \\
$120-480$ & 0,040 & 0,086 & 0,016 & 0,976 & $-4,733$ & 2,1 \\
$480-1440$ & 1,136 & 2,063 & 0,963 & 0,088 & 1,217 & 2,6 \\
$5-1440$ & 0,157 & 0,308 & 0,390 & 0,336 & 0,163 & 4,8 \\
\hline
\end{tabular}

Com essas equações ajustadas pode-se estimar a chuva para qualquer duração entre 5 min e 24 h e período de retorno de 2 a 100 anos, a partir da chuva $\mathrm{P}_{10}{ }^{60}$, que deve ser previamente conhecida.

Nota-se, na Figura 1, que as relações obtidas para Urussanga, SC, apresentam valores próximos aos obtidos com as equações ajustadas por Bell (1969) e Uehara et al. (1980) para o Brasil e São Paulo, com diferenças inferiores a $10 \%$ exceto na duração de 5 min quando a estimativa obtida para Urussanga apresenta valores em torno de 20\% menores.

Segundo Costa \& Rodrigues (1999), a metodologia das isozonas, proposta por Torrico (1975) pode levar a desvios da ordem de 7,5 a 54\% em relação às equações IDF. Oliveira et al. (2000), comparando as estimativas de chuvas obtidas por desagregação da chuva pelas relações da CETESB (1986) com as equações IDF obtidas a partir dos dados do pluviógrafo, encontraram erros relativos abaixo de 15\%. Genovez et al. (1994) compararam cinco equações IDF generalizadas, incluindo a proposta por Bell (1969) e por Uehara et al. (1980) para o Brasil e São Paulo, com dados de 9 postos pluviográficos do Estado de São Paulo e constataram que as equações IDF generalizadas apresentaram resultados semelhantes e, de maneira geral, os desvios absolutos dos valores estimados pelas equações com os observados, foram inferiores a $15 \%$, embora para alguns postos este desvio tenha chegado a 45\%. Damé et al. (2006), analisando dados de Pelotas, também observaram estimativas semelhantes para as intensidades de chuva estimadas com as equações IDF e pelo método das relações entre durações.

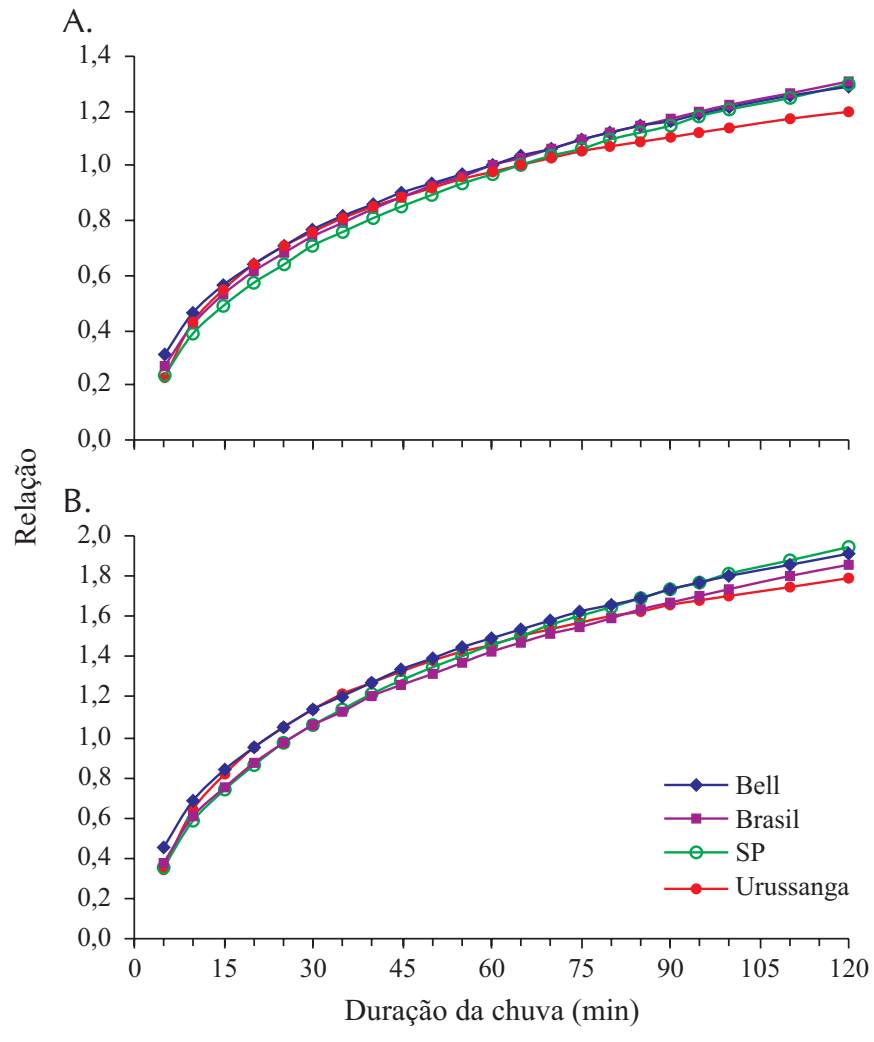

Figura 1. Relação entre a chuva com período de retorno de 10 anos (A) e de 100 anos (B) e duração de até 120 min e a chuva com período de retorno de 10 anos e duração de $60 \mathrm{~min}$

Tem-se, na Figura 2, as relações entre a chuva de duração t min e período de retorno variando de 2 a 100 anos com a chuva $\mathrm{P}_{10}{ }^{60}$; observa-se, na Figura $2 \mathrm{~A}$, que para a duração de 60 min a relação varia de 0,6 para o período de retorno de 2 anos até 1,45 para o período de retorno de 100 anos, com grande variação nos valores dessas relações, em função da duração da chuva; já para as durações entre 120 e 480 min (Figura 2B) e entre 480 e 1440 min (Figura 2C) a menor variação nas relações, com comportamento aproximadamente linear.

Apresentam-se, na Tabela 3, os coeficientes da equação de Bell, substituindo a chuva $\mathrm{P}_{10}{ }^{60}$ pela chuva com período de retorno de 10 anos e duração de 1 dia $\left(\mathrm{P}_{10}{ }^{1 \mathrm{dia}}\right)$. Obtém-se, através dos valores do erro padrão de estimativa, a mesma precisão na estimativa da chuva, indicando que essas equações podem ser usadas nos locais que dispõem apenas das informações de chuva com duração de 1 dia.

Tabela 3. Coeficientes da equação de Bell em função da chuva com período de retorno de 10 anos e duração de 1 dia $\left(\mathrm{P}_{10}{ }^{1 \text { dia }}\right)$

\begin{tabular}{ccccccc}
\hline $\begin{array}{c}\text { Duração } \\
\text { (min) }\end{array}$ & a & b & c & d & e & $\begin{array}{c}\text { Erro padrão } \\
\text { (mm) }\end{array}$ \\
$5-120$ & 0,797 & 1,890 & 0,887 & 0,033 & 0,911 & 2,2 \\
$120-480$ & 0,025 & 0,053 & 0,010 & 0,977 & $-2,992$ & 2,1 \\
$480-1440$ & 1,372 & 2,491 & 0,403 & 0,075 & 0,497 & 2,6 \\
$5-1440$ & 0,102 & 0,199 & 0,241 & 0,334 & 0,107 & 4,8 \\
\hline
\end{tabular}


A.

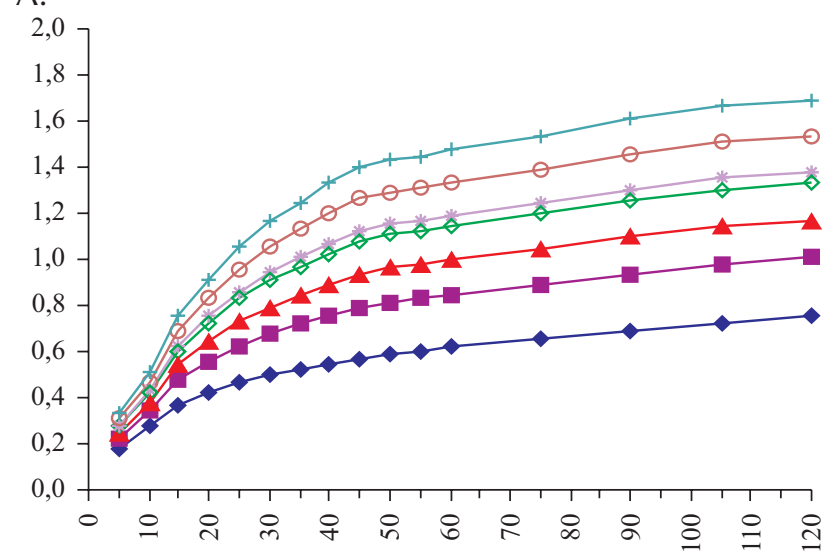

B.

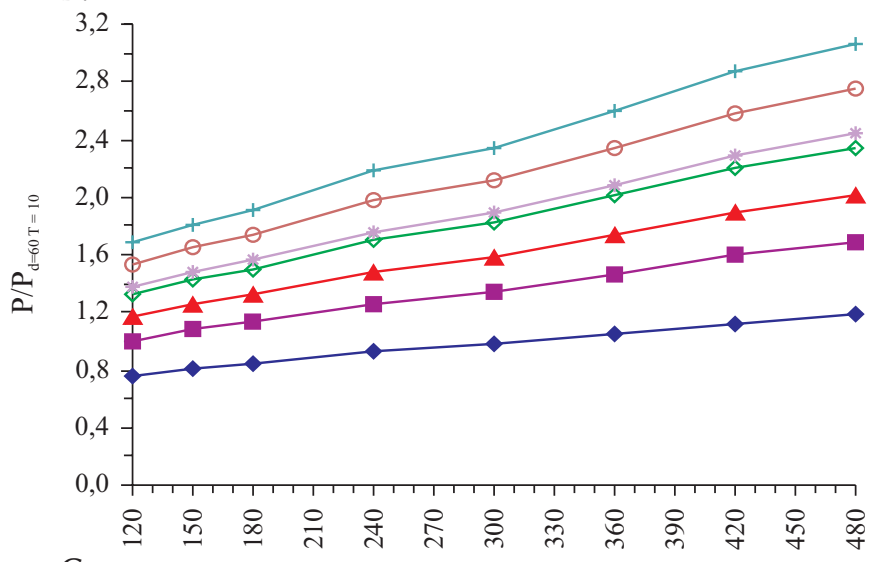
C.

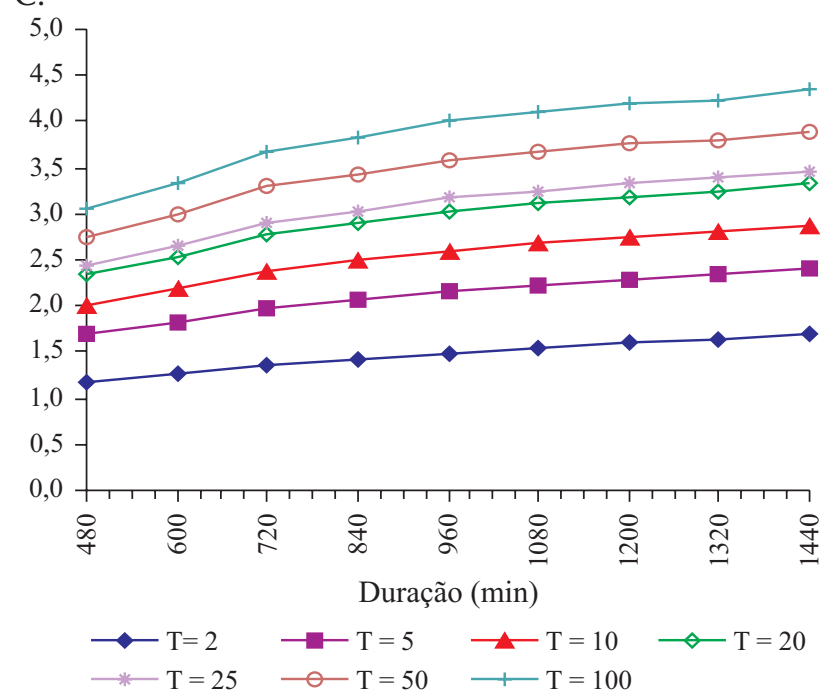

Figura 2. Relações entre chuva de duração de 5 a 120 min (A), de 120 a 480 min (B) e de 480 a 1440 min (C) e a chuva com período de retorno de 10 anos e duração de $60 \mathrm{~min}$

As relações entre chuvas de diferentes durações estão na Figura 3. Na Figura 3A observa-se que a precipitação com duração de 60 min e o período de retorno de 10 anos apresenta relação 0,392 indicando 39,2\% da chuva máxima diária com período de retorno de 10 anos. O comportamento das curvas da Figura 3 é semelhante aos da Figura 2, diferindo apenas na magnitude das relações.

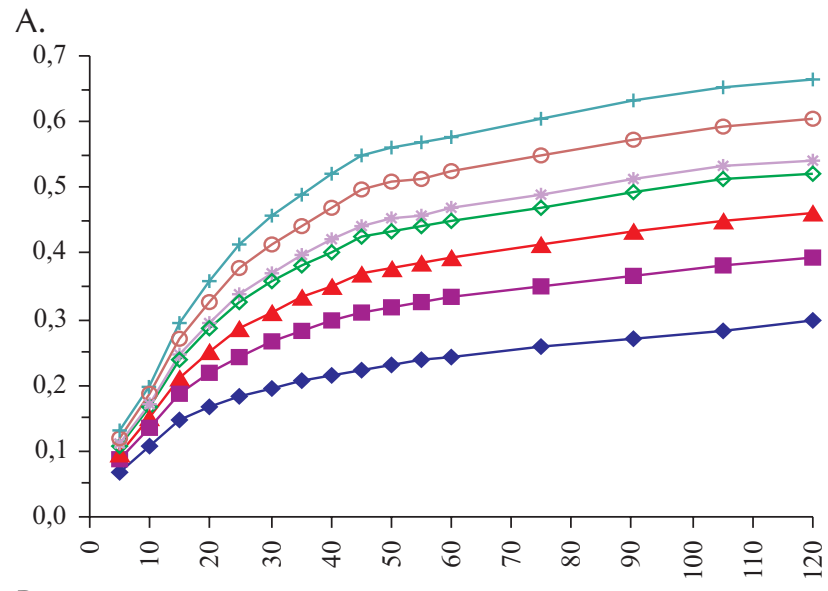

B.
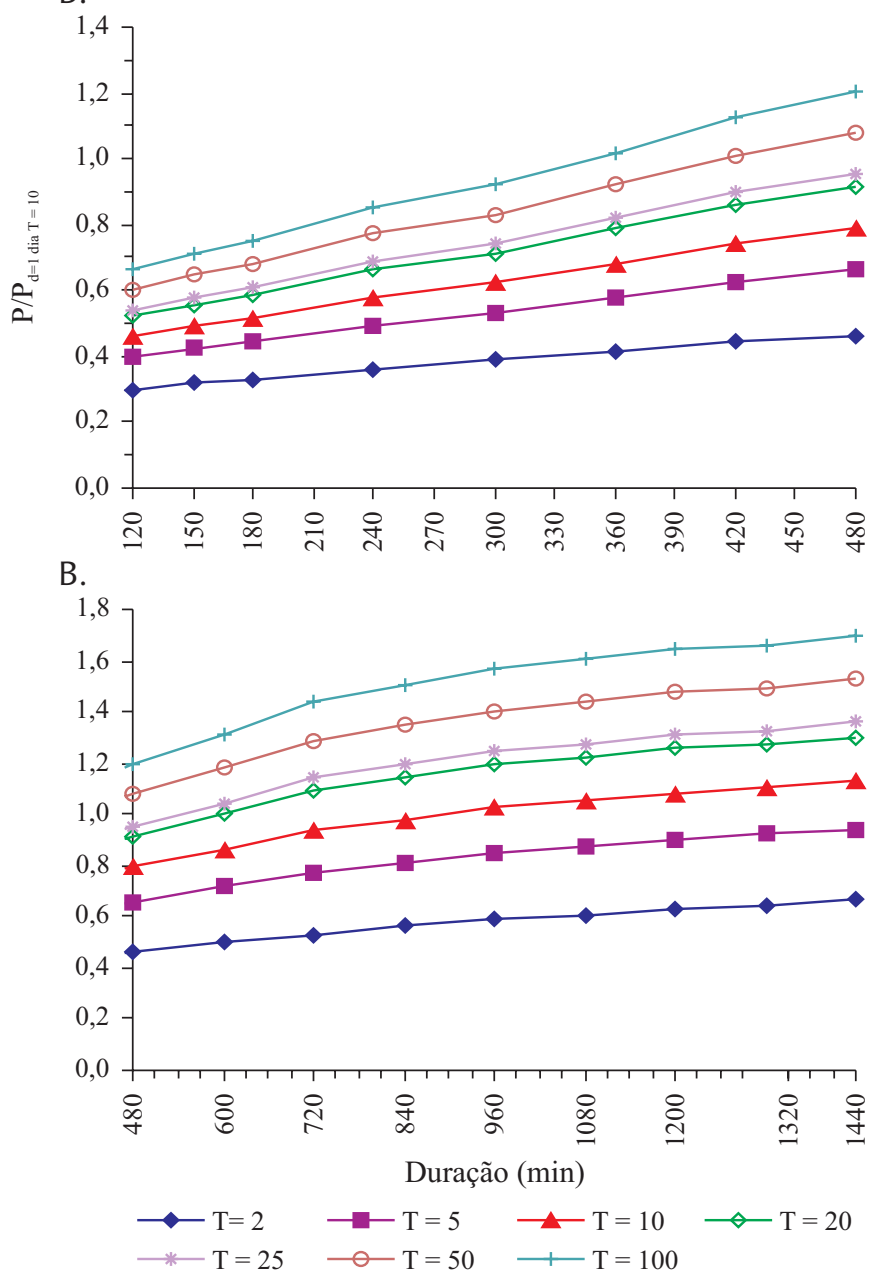

Figura 3. Relações entre chuva de duração de 5 a 120 min (A), de 120 a 480 min (B) e de 480 a 1440 min (C) e a chuva com período de retorno de 10 anos e duração de 1 dia

\section{CONCLUSÕES}

1. As relações entre precipitações intensas de duração entre 10 e 120 min obtidas para Urussanga, apresentam diferenças inferiores a 10\% das relações obtidas por Bell (1969) e das relações indicadas por Uehara et al (1980).

2. A relação média da precipitação máxima de 24 h e da precipitação máxima de 1dia, é de 1,13. 
3. As relações obtidas com base na chuva máxima com duração de um dia e período de retorno de 10 anos, apresentam erro padrão de estimativa equivalente às obtidas com base na chuva com duração de 1 h e período de retorno de 10 anos, além de alternativa para os locais sem registro de dados pluviográficos.

\section{LITERATURA CITADA}

Back, A. J. Chuvas intensas e chuva de projeto de drenagem superficial para o estado de Santa Catarina. Florianópolis: EPAGRI, 2002. 65p.

Bell, F. G. Generalized rainfall-duration-frequency relationships. Journal of Hydraulics Division -ASCE, v.95, p311-327, 1969.

CETESB - Companhia de Tecnologia de Saneamento Ambiental. Drenagem urbana - manual de projeto. 3.ed. São Paulo: CETESB, 1986. 464p.

Costa, A. R.; Brito, V. F. Equações de chuva intensa para Goiás e Sul de Tocantins. In: Simpósio Brasileiro de Recursos Hídricos, 13, 1999, Belo Horizonte. Anais... Belo Horizonte: ABRH, 1999. CD Rom

Costa, A. R.; Rodrigues, A. A. Método das isozonas: desvios entre resultados In: Simpósio Brasileiro de Recursos Hídricos, 13, 1999, Belo Horizonte. Anais... Belo Horizonte: ABRH, 1999. CD Rom

Damé, R. C. F. Desagregação de precipitação diária para estimativa de curvas Intensidade-Duração-Frequência. Porto Alegre: UFRGS, 2001. 131p. Tese Doutorado

Damé, R. C. F.; Pedrotti, C. B. M.; Cardoso, M. A.; Silveira, C. P.; Duarte, L. A.; Moreira, A. C. Comparação entre curvas intensidade-Duração-Frequência de ocorrência de precipitação obtidas a partir de dados pluviográficos com aquelas estimadas por técnicas de desagregação de chuva diária. Revista Brasileira de Agrociência, v.12, n.4, p.505-509, 2006.

Damé, R. C. F.; Viegas Filho, J. S.; Godinho, G. S. E. Desagregação de precipitação diária mediante o uso da modelagem de precipitação e método das relações para estimar as curvas IDF. In: Congresso Brasileiro de Engenharia Agrícola, 31, 2003, Goiânia. Anais... Goiânia: SBEA, 2003. CD Rom.

Froehlich, D. C. Short-duration-rainfall intensity equations for drainage design. Journal of Irrigation and Drainage Engineering, v.119, n.5, p.814-828. 1993.

Fendrich, R. Chuvas intensas para obras de drenagem no Estado do Paraná. Curitiba: Champagnat, 1998. 99p.

Genovez, A. M.; Pegoraro, R. C. F. Análise e avaliação de equações de chuvas intensas generalizadas. In: Congresso Latinoamericano de Hidráulica, 18, 1998, Oaxaca. Anais... Oaxaca: IAHR, v.1, 1998. p.369-79.

Genovez, A. M.; Zuffo, A. C.; Borri Genovez, A. I. Relação entre chuvas intensas de diferente duração e avaliação das equações de chuvas generalizadas. In: Congresso Latinoamericano de Hidráulica, 16, 1994, Santiago. Anais... Santiago: IAHR, v.3. p.279-90. 1994.
Kessler, J.; Raad, S. J. Análise de dados pluviométricos. In: International Institute for Land Reclamation and Improvement. Principios y aplicaciones del drenaje. Wageningen: ILRI. v.3, p.16-5, 1978. Publication 16.

Kite, G. W. Frequency and risk analysis in hydrology. Fort Collins: Water Resources. 3.ed. 1978. 224p.

Martinez Junior, F. Análise das precipitações intensas no Estado de São Paulo. In: Simpósio Brasileiro de Recursos Hídricos, 13, 1999, Belo Horizonte. Anais... Belo Horizonte: ABRH, 1999. CD-Rom

Mello, C. R.; Silvar, A. M.; Lima, J. M.; Ferreira, D. F.; Oliveira, M. S. Modelos matemáticos para predição da chuva de projeto para regiões do Estado de Minas Gerais. Revista Brasileira de Engenharia Agrícola e Ambiental, v.7, n.1, p.121128, 2003.

Nerilo, N.; Medeiros, P. A.; Cordeiro, A. Chuvas intensas no Estado de Santa Catarina. Florianópolis: UFSC/Edifurb, 2002. 156p.

Occhipinti, A. G.; Santos, P. M. Análises das máximas intensidades de chuva na cidade de São Paulo. São Paulo: USP, Instituto Agronômico e Geofísico, 1965. 40p.

Occhipinti, A. G.; Santos, P.M. Relações entre as precipitações máximas de um dia e de 24 horas na cidade de São Paulo. São Paulo: IAU/USP, 1966. 10p.

Oliveira. L. F.; Cortes, F. C.; Barbosa, F. O. A.; Romão, P. A. Carvalho, D. F. Estimativa das equações de chuvas intensas para algumas localidades de Goiás pelo método da desagregação de chuvas. Pesquisa Agropecuária Tropical, v.30, n.1, p.23-27, 2000.

Pinto, F. A.; Ferreira, P. A.; Pruski, F. F.; Alves, A. R.; Cecon, P. R. Equações de chuvas intensas para algumas localidades do Estado de Minas Gerais. Engenharia Agrícola, v.16, n.1, p.91-104, 1996.

Silva, D. D.; Gomes Filho, R. R.; Pruski, F. F.; Pereira, S. B.; Novaes, L. F. Chuvas intensas no Estado da Bahia. Revista Brasileira de Engenharia Agrícola e Ambiental, v.6, n.2, p.362-367, 2002.

Silva, D. D.; Pinto, F. R. L. P.; Pruski, F. F.; Pinto, F. A. Estimativa e espacialização dos parâmetros da equação de intensidade-duração-frequência da precipitação para os Estados do Rio de Janeiro e Espírito Santo. Revista de Engenharia Agrícola, v.18, n.3, p.11-21, 1999.

Souza, F. A. S.; Silva, V. P. R. Gestão e controle ambiental. Revista Brasileira de Engenharia Agrícola e Ambiental, v.2, n.2, p.319-323, 1998.

Torrico, J. J. T. Práticas hidrológicas. Rio de Janeiro: Transcon, 1975. 119p.

Uehara, K.; Zahed Filho, K.; Silveira, L. N. L.; Palermo, M. A. Pequenas bacias hidrográficas do Estado de São Paulo: Estudo de vazões médias e máximas. São Paulo: FDTE/Escola Politécnica da USP, v. 2, 1980. 780p.

Weiss, L. L. Ratio of true to fixed-interval maximum rainfall. Journal of Hydraulics Division, v. 90, p.77-82, 1964. 